Journal of Social Sciences 7 (3): 354-357, 2011

ISSN 1549-3652

(C) 2011 Science Publications

\title{
Tensiomyographic Characteristics of Rectus Femoris after a Single Bout of Intense Exercise
}

\author{
${ }^{1}$ Luis Carrasco Paez, ${ }^{1}$ Borja Sañudo Corrales and \\ ${ }^{1}$ Moises de Hoyo Lora, ${ }^{2}$ Gabriela Ochiana \\ ${ }^{1}$ Department of Physical Education and Sport, University of Seville, Spain \\ ${ }^{2}$ Department of Sports and Human Movement Sciences, Faculty of Health, \\ University "Vasile Alecsandri" of Bacau, Romania
}

\begin{abstract}
Problem statement: It is not well known how local fatigue can impair the contractile properties of skeletal muscle, so the aim of this study was to determine the Tensiomyographic (TMG) characteristics of Rectus Femoris (RF) after a single bout of intense exercise. Approach: Twelve healthy male volunteers (mean \pm SEM; age, $24.16 \pm 0.62$ years, height $172.02 \pm 1.21 \mathrm{~cm}$, body mass $71.84 \pm 2.19 \mathrm{~kg}$, BMI $24.42 \pm 0.70 \mathrm{~kg} \mathrm{~m}^{-1}$ ) were recruited. Results: In session 1, a graded exercise test on cycle ergometer was performed in order to determine subject's maximal oxygen uptake $\left(\mathrm{VO}_{2 \max }\right)$ and power at this key point $\left(\mathrm{pVO}_{2 \max }\right)$. In a second testing session, TMG rest values were assessed and then all subjects carried out 2 min of cycling at $\mathrm{pVO}_{2 \max }$ after which TMG analysis was applied again. The results showed subject's $\mathrm{VO}_{2 \max }$ values of $53.44 \pm 2.67 \mathrm{ml} \mathrm{kg}^{-1} \mathrm{~min}^{-1}$, reaching a mean $\mathrm{pVO}_{2 \max }$ of $270.83 \pm 8.61 \mathrm{~W}$. Maximal radial displacement $(\mathrm{Dm})$ and time from the onset of electrical stimulus to $10 \%$ of Dm (Td) were significantly lower at post-test (from 7.577.57 $\pm 0.92-4.37 \pm 0.67 \mathrm{~mm} ; \mathrm{p}=0.01$, for Dm and from 25.25 $\pm 0.57-22.44 \pm 0.89 \mathrm{~ms} ; \mathrm{p} \leq 0.05$, for Td). Conclusion/Recommendations: It can be concluded that a brief and intense exercise consisting in 2 min of cycling at $\mathrm{PVO}_{2 \max }$ provokes a reduction in contractile properties of RF considering TMG parameters such as Dm and Td. More studies are needed to clarify the role of muscle fatigue on temporal TMG parameters.
\end{abstract}

Key words: Intense exercise, Tensiomyography (TMG,), maximal radial displacement, radial displacement, muscle's belly, self-adhesive, contractile parameters, Rectus Femoris (RF)

\section{INTRODUCTION}

Tensiomyography (TMG) is a relatively novel technique based on the quantification of radial muscle belly displacement in response to a single electrical stimulus. In this way, the basis of TMG is focused on enlargement of muscle's belly during its contraction. Using a specific displacement sensor, the radial enlargement of the muscle belly can be measured, drawing a displacement-time curve which allows analyzing the muscle contractile properties from different parameters (Valencic and Knez, 1997). These parameters are: Maximal radial Displacement (Dm), time from the onset of electrical stimulus to $10 \%$ of $\mathrm{Dm}(\mathrm{Td})$, time from $10-90 \%$ of $\mathrm{Dm}$ in the ascending curve (Tc), time between $50 \%$ of Dm on both sides of the curve (Ts) and time from $90-50 \%$ of Dm on the descending curve (Tr) (Fig. 1). Recently, reliability of TMG has been established, reaching a good to excellent inter-rater reliability level for practically all contractile parameters (Tous-Fajardo et al., 2010; Carrasco et al., 2011).

In the last few years, measurements of contraction time by TMG have been successfully implemented on different muscle groups (Valencic and Knez, 1997) to investigate endurance and muscle belly stiffness among other muscular responses (Grabljevec et al., 2005; Pisot et al., 2008). However, muscle contractile properties during or after fatiguing exercise remains unclear (Letafatkar et al., 2009; Hosseinimehr et al., 2010) mainly due to a lack of adequate assay system. Thus, the purpose of this study was to evaluate the TMG responses of exercised muscles after a single bout of intense exercise.

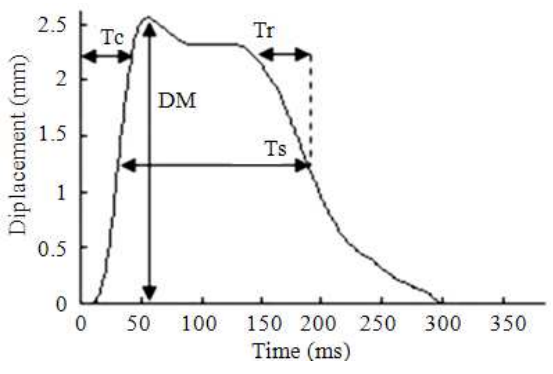

Fig. 1: Graphical representation of some TMG parameters 


\section{MATERIALS AND METHODS}

Participants: Twelve healthy male volunteers (mean \pm SEM; age, $24.16 \pm 0.62$ years, height $172.02 \pm 1.21 \mathrm{~cm}$, body mass $71.84 \pm 2.19 \mathrm{~kg}$, BMI $24.42 \pm 0.70 \mathrm{~kg} \cdot \mathrm{m}^{-2}$ ) were recruited for this study. All were recreationally active who were not engaged in a systematic exercise programmed. Participants were asked to abstain for food and caffeine beverages for $2 \mathrm{~h}$ before testing and to not perform heavy exercise during the $24 \mathrm{~h}$ preceding the tests. After being fully informed about the purpose of the experiments, each participant signed an informed consent. The Local Research Ethical Committee approved the experimental protocol and the procedures involved.

Testing procedures: All participants reported to laboratory for two visits. The first one was dedicated to determine participants' $\mathrm{VO}_{2 \max }$ and power associated with $\mathrm{VO}_{2 \max }\left(\mathrm{pVO}_{2 \max }\right)$; for that, the participants carried out a maximal graded test on a cycle ergometer. Then, on a separate session $(48 \mathrm{~h})$ and both before and after $2 \mathrm{~min}$ of cycling at $\mathrm{pVO}_{2 \max }$ (intense exercise) TMG measures were assessed. All testing procedures were performed at the same hour interval (between 9:00-11:00 am) in a room at an ambient temperature of $22-24{ }^{\circ} \mathrm{C}$.

Maximal graded exercise test: After a standardized warm-up consisting of $5 \mathrm{~min}$ of pedaling on a cycle ergometer (Kettler Axiom P2, GmbH and Co.KG, Ense-Parsit, Germany) at a load of $50 \mathrm{~W}$, participants performed a maximal graded exercise test. The initial load was $50 \mathrm{~W}$ and increased by $25 \mathrm{~W}$ every min until exhaustion with a pedaling frequency of $60 \mathrm{rpm}$. Gas analyses were performed using a breath-by-breath gas analyzer (VO2000 Portable Metabolic System, Medgraphics, St. Paul, MN), whose validity was previously proved by Byard and Dengel (2002). Before each test VO2000 was manually calibrated according to manufacturer guidelines. $\mathrm{VO}_{2 \max }$ corresponded to the highest $\mathrm{VO}_{2}$ attained in two successive $15 \mathrm{~s}$ periods for the maximal graded test. In addition, HR was continuously monitored (Polar $800 \mathrm{CX}$, Polar Electro, Kempele, Finland). It was considered that participants had reach their $\mathrm{VO}_{2 \max }$ when three or more of the following criteria were met: (a) a steady state of $\mathrm{VO}_{2}$ despite increasing load (changes in $\mathrm{VO}_{2}$ at $\mathrm{VO}_{2 \max } \leq 150$ $\mathrm{mL}$ ); (b) a final respiratory exchange ratio higher than 1:1; (c) visible exhaustion; (d) an HR at the end of the exercise $\left(\mathrm{HR}_{\max }\right)$ within the $10 \mathrm{bpm}$ of the predicted maximum (220-age). Also, $\mathrm{pVO}_{2 \max }$ was defined as power output (W) developed by the participants on the cycle ergometer when $\mathrm{VO}_{2 \max }$ was reached.

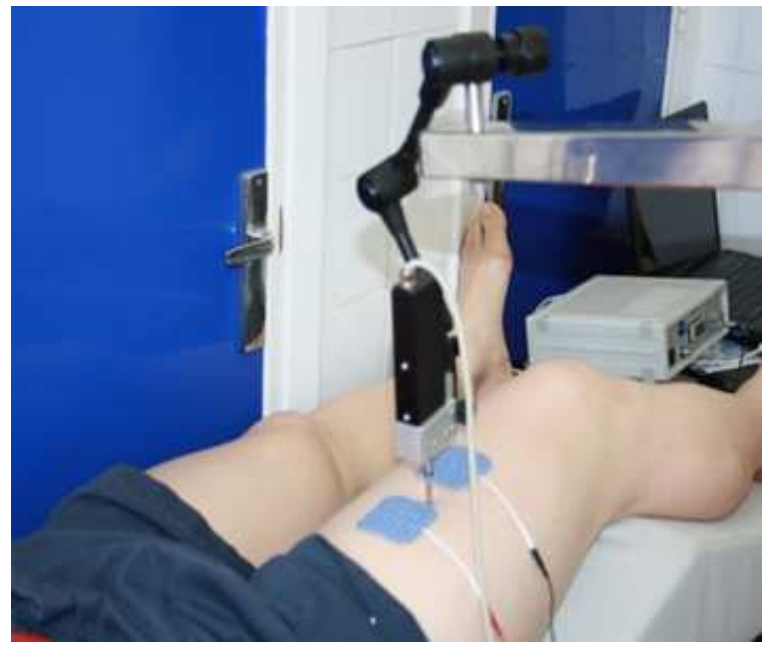

Fig. 2: TMG assessment in RF of right leg

Intense exercise and TMG measurement: In session 2 , the participants performed $2 \mathrm{~min}$ of cycling at $\mathrm{pVO}_{2 \max }$ keeping a pedaling frequency of $60 \mathrm{rpm}$. In our study, before (pre) and after intense exercise (post), tensiomyography analysis of Rectus Femoris (RF) of the right leg was performed. Radial displacements were measured under static and relaxed conditions, with the participant in the supine position and the knee joint fixed at a 120 angle (180 corresponding to full extension of the knee). The measured limb was positioned on a triangular wedge foam cushion to keep a fixed knee angle. A digital displacement transducer (GK 40, Panoptik d.o.o., Ljubljana, Slovenia), which incorporates a spring of $0.17 \mathrm{~N} \cdot \mathrm{mm}^{-1}$, was set perpendicular to the muscle belly to acquire RF radial displacement. Sensor location was determined anatomically according to Delagi et al. (1975) and marked with a dermatological pen. Two square $(5 \times 5 \mathrm{~cm}) 2 \mathrm{~mm}$ thick self-adhesive electrodes (Compex Medical SA, Ecublens, Switzerland) were placed symmetrically $5 \mathrm{~cm}( \pm 5 \mathrm{~cm})$ distal and proximal to the sensor tip. A TMG-S1 (EMF-Furlan and Co. d.o.o., Ljubljana, Slovenia) stimulator was used (Fig. 2).

Regarding electrical stimulation procedures, pulse duration was $1 \mathrm{~ms}$ and the initial current amplitude was $30 \mathrm{~mA}$. For each of the TMG assessment, current amplitude was progressively increased by $10 \mathrm{~mA}$ increments until there was no further increase in Dm or maximal stimulator output $(110 \mathrm{~mA})$. Rest periods of 15 were interspersed between consecutive measurements to minimize the effects of fatigue and 
potentiation. For each participant, two consecutive measurements were performed at the highest current amplitude and they were recorded and averaged for subsequent analyses. In any case, none of the participants reported discomfort during electrical stimulation.

In the present study Dm, Ts and $\mathrm{Tr}$ were registered so these parameters seem to show largest influence to muscle fatigue rate and are also expected to be the best measure of the fatigue rate (Krizaj et al., 2008).

Statistical analyses: Means and standard errors of the mean were calculated for each variable. Normality assumption was checked using the KolmogorovSmirnov test and all variables were normally distributed. One way analysis of variance was used to compare pre- and post-values Moreover. Eta-squared $\left(\eta^{2}\right)$ was used for the calculation of effect size. Statistical significance was set at $\mathrm{p} \leq 0.05$.

\section{RESULTS}

In session 1, mean $\mathrm{VO}_{2 \max }$ measured during maximal graded exercise test was $53.44 \pm 2.67$ $\mathrm{mL} \cdot \mathrm{kg}^{-1} \cdot \mathrm{min}^{-1}$, reaching a mean $\mathrm{pVO}_{2 \max }$ of $270.83 \pm$ $8.61 \mathrm{~W}$.

Table 1 shows TMG data obtained before and after intense exercise. The mean electric stimulus applied to RF before intense exercise was $98.89 \pm 4.60 \mathrm{~mA}$, similar to that applied just at the end of exercise. It is important to note that only $\mathrm{Dm}$ and $\mathrm{Td}$ were significantly lower at post-test (from 7.577.57 \pm 0.92 $4.37 \pm 0.67 \mathrm{~mm} ; \mathrm{p}=0.01$, for Dm and from $25.25 \pm$ 0.57-22.44 $\pm 0.89 \mathrm{~ms} ; \mathrm{p} \leq 0.05$, for $\mathrm{Td}$ ), showing also small-moderate effect sizes by attending to the Cohen's effect size criteria (Cohen, 1988). On the other hand, Ts and $\operatorname{Tr}$ didn't show significant alterations, although TS increased from $141.06 \pm$ 18.01-165.32 $\pm 20.79 \mathrm{~ms}$ and $\operatorname{Tr}$ decreased lightly (from $54.84 \pm 6.37-52.73 \pm 8.28 \mathrm{~ms}$ ).

Table 1: TMG assessment

\begin{tabular}{llllll}
\hline & Pre-test & Post-test & F & sig & $\eta^{2}$ \\
\hline Dm (mm) & $7.57 \pm 0.92$ & $4.37 \pm 0.67$ & 7.930 & 0.010 & 0.265 \\
Ts (ms) & $141.06 \pm 18.01$ & $165.32 \pm 0.79$ & 0.770 & 0.390 & 0.034 \\
Tr (ms) & $54.84 \pm 6.37$ & $52.73 \pm 8.28$ & 0.041 & 0.842 & 0.002 \\
Td (ms) & $25.25 \pm 0.57$ & $22.44 \pm 0.89$ & 7.088 & 0.014 & 0.244
\end{tabular}

Data are expressed as mean \pm SEM. Pre-test: before intense exercise; Post-test: after intense exercise; F, sig and $\eta^{2}$ (eta-squared) values are related to one-way ANOVA analysis

\section{DISCUSSION}

An innovation of the present study is the use of TMG to evaluate the effects of intense cycling on contractile properties of exercised muscles. From all TMG parameters, Dm, Ts and Tr seem to provide the most useful information in evaluation of muscle fatigue (Krizaj et al., 2008). Dm, which could be equated to electrically evoked peak twitch torque, is determined by both the number and the type of muscle fibers recruited by the electrical stimulus, being considered as a measure of muscle belly stiffness (Pisot et al., 2008). In the present study TMG analysis of RF of right leg was performed before (pre-test) and after intense exercise (post-test), reported significant decreases in Dm and Td values, although no statistical differences were observed when $\operatorname{Tr}$ and Ts assessed in pre-test and posttest were compared. Decreases in Dm and Td after intense exercise could indicate a lower contractile capacity of RF due to a fatigue state, that is to say, a lower enlargement of muscle's belly during its contraction and a slower reaction after electrical stimulus, respectively. However, it seem difficult to explain RF fatigue from $\operatorname{Tr}$ (time from $90-50 \%$ of Dm on the descending curve) and $\mathrm{Td}$ (time from the onset of electrical stimulus to $10 \%$ of Dm) values observed in post-test assessment, since under fatigue state these data should be higher compared to those obtained in resting conditions.

\section{CONCLUSION}

Brief and intense exercise consisting in 2 min of cycling at $\mathrm{VO}_{2 \max }$ power output provokes a reduction in contractile properties of RF muscle considering TMG parameters such as Dm and Td. However, more studies are needed to clarify how muscle fatigue can affect to temporal TMG parameters related to muscle contraction maintenance and relaxation.

\section{REFERENCES}

Byard, A.D. and D.R. Dengel, 2002. Validity of a portable metabolic measurement system. Med. Sci. Sports Exerc., 34: S149-S149. DOI: 10.1097/00005768-200205001-00829

Cohen, J., 1988. Statistical Power Analysis for the Behavioral Sciences. 2nd Edn., Routledge, London, ISBN: 0805802835, pp: 567.

Delagi, E.F., 1975. Anatomic Guide for the Electromyographer: The Limbs. 1st Edn., C.C. Thomas, Springfield, ISBN: 0398032955, pp: 207. 
Grabljevec, K., H. Burger, K. Kersevan, V. Valencic and C. Marincek, 2005. Strength and endurance of knee extensors in subjects after paralytic poliomyelitis. Disabil. Rehabil., 27: 701-799. DOI: 10.1080/09638280400020623

Hosseinimehr, S.H., H. Daneshmandi and A.A. Norasteh, 2010. The Effects of Fatigue and Chronic Ankle Instability on Dynamic Postural Control. Phys. Int. 1: 22-26. DOI: 10.3844/PISP.2010.22.26

Krizaj, D., B. Simunic and T. Zagar, 2008. Short-term repeatability of parameters extracted from radial displacement of muscle belly. Elect. Kinesiol., 18: 645-651. DOI: 10.1016/J.JELEKIN.2007.01.008 PMid:17379538

Letafatkar, K., M.H.Alizadeh, M.R. Kordi, 2009. The Effect of Exhausting Exercise Induced Fatigue on the Double-Leg Balance of Elite Male Athletes. J. Soc. Sci., 5: 445-451 DOI: 10.3844/JSSP.2009.445.451
Pisot, R., M.V. Narici, B. Simunic, M. De Boer and O. Seynnes, et al., 2008. Whole muscle contractile parameters and thickness loss during 35-day bed rest. Eur. J. Appl. Phys., 104: 409-14. DOI: 10.1007/S00421-008-0698-6 PMid:18297302

Tous-Fajardo, J., G. Moras, S.R. Jimenez, R. Usach and D. Moreno et al., 2010. Inter-rater reliability of muscle contractile property measurements using non-invasive tensiomyography. J. Elect. Kinesiol., 20:

761-766.

DOI:10.1016/J.JELEKIN.2010.02.008 PMid: 20236839

Valencic, V. and N. Knez, 1997. Measuring of skeletal muscles' dynamic properties. Artif. Organs, 21: 240-242. DOI: $\quad 10.1111 /$ J.15251594.1997.TB04658.X 